\title{
A fast 4-channel silicon switch using an AWG with 12 carrier depletion modulators
}

\author{
Shibnath Pathak ${ }^{* \dagger}$, Hui Yu* ${ }^{* \ddagger}$ Dries Van Thourhout*, Wim Bogaerts*§, \\ *Photonics Research Group, Ghent University - imec,Department of Information Technology \\ Center of Nano- and Biophotonics (NB-photonics), Ghent, Belgium \\ $\dagger_{n}$ now with: University of California Davis, Dept. of Electrical and Computer Engineering, Davis, CA 95616, USA \\ ${ }^{\ddagger}$ now with: Zhejiang University, Dept. of Information Science and Electronic Engineering, Hangzhou 310027, China

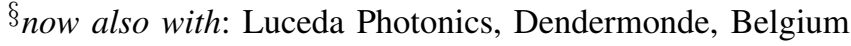

\begin{abstract}
We demonstrate a $1 \times 4$ optical phased array based switch in silicon-on-insulator using 12 interdigitated carrierdepletion based silicon modulators. The device footprint is $\mathbf{3 . 3 9}$ $\times 0.57 \mathrm{~mm}^{2}$ using $3.2 \mathrm{~mm}$ long phase modulators. The measured insertion loss is $-6.0 \mathrm{~dB}$ and the crosstalk is better than $-13 \mathrm{~dB}$.
\end{abstract}

\section{INTRODUCTION}

Optical Packet Switching can be a solution for efficient use of optical networks, but this requires switch fabrics with fast reconfiguration. Different schemes have been proposed, but not all scale to large channel counts without severe loss and crosstalk penalty. The single-stage modulation scheme seems a good choice for a large channel integrated switch [1].

We implement a 4-channel single-stage modulation switch using an AWG, as shown in Fig. 1. This consists of a zeroorder phased array with individual phase modulators in each arm. The grating will refocus the light from input to the output in the same position, and this for all wavelengths. Now to shift the light from one output to another a phase shift is applied with the electro-optical phase shifters. For high-speed switching performance we use carrier depletion modulators.

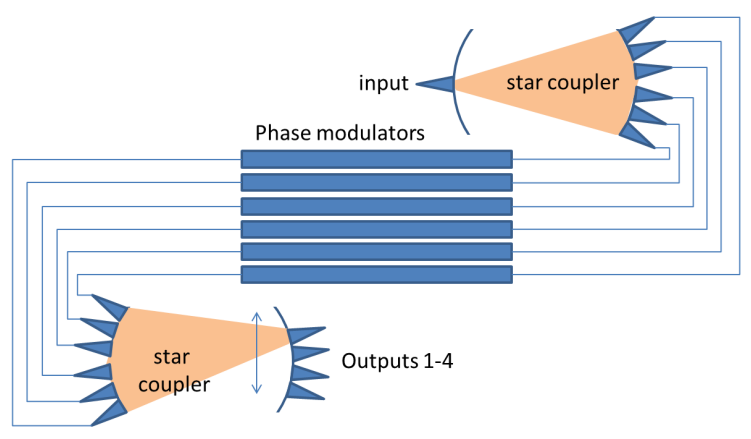

Fig. 1. Optical phased array switch based on single stage modulation.

\section{DESIGN}

\section{A. Carrier-depletion based silicon modulators}

For a switch, each phase modulator should cover a full $2 \pi$ phase change. Also the optical loss should be as low as possible and the operation speed sufficiently high for switching. We use carrier-depletion modulators with an interdigitated pn junction in the core of the waveguide. This junction has a lower operation speed due to its higher RC constant, but it has a higher modulation efficiency with an interdigitated junction [2]. This modulator has a modulation efficiency $V_{\pi} \cdot L_{\pi}$ of approximately $1.5 \mathrm{~V} . \mathrm{cm}$. As the diode reaches its breakdown at $-9 \mathrm{~V}$ reverse bias, we need to choose the modulator length such that we get at least $2 \pi$ phase change within this voltage limit. Taking sufficient margin we used $3.2 \mathrm{~mm}$ long modulators. The estimated insertion loss of this modulator is $-6 \mathrm{~dB}$.

\section{B. Phased array}

A zeroth order AWG requires an S-shaped layout, shown in Fig. 1. The waveguides contain equal-length modulators, while the other sections consist of $450 \mathrm{~nm}$ bends and $800 \mathrm{~nm}$ straight waveguides with individually balanced path lengths.

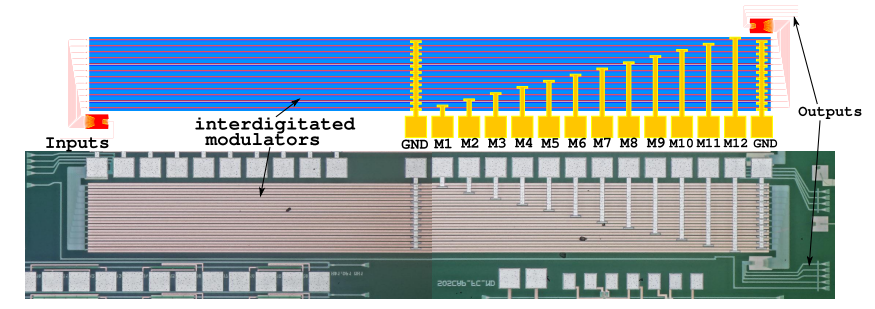

Fig. 2. The $1 \times 4$ switch with 12 modulated arms.

We designed a $1 \times 4$ optical phased array switch with 12 arms. The number of arms (and therefore the number of channels) in our case was limited by the test and measurement equipment (i.e. the number of modulators we can drive simultaneously, and in this case only at low speed). The low number of arms will result in a less steep roll-off between the output ports, and therefore a non-negligible neighbor channel crosstalk. This is, in turn, expressed as a smaller extinction ratio. One can increase the extinction by using more modulated arms in the array. The interface with the waveguide array and the slab is shallow-etched to avoid reflection [3].

\section{SimUlation}

The simulation is performed using the integrated design and simulation framework IPKISS [4]. We adapted the AWG simulation to include a variable phase modulation in the arms. As all the waveguides are equally long the spectral response will be almost wavelength independent. To find the transmission variation with respect to the phase introduced by the modulators we run a loop of simulations while the phase is varying from $-2 \pi$ to $2 \pi$. 


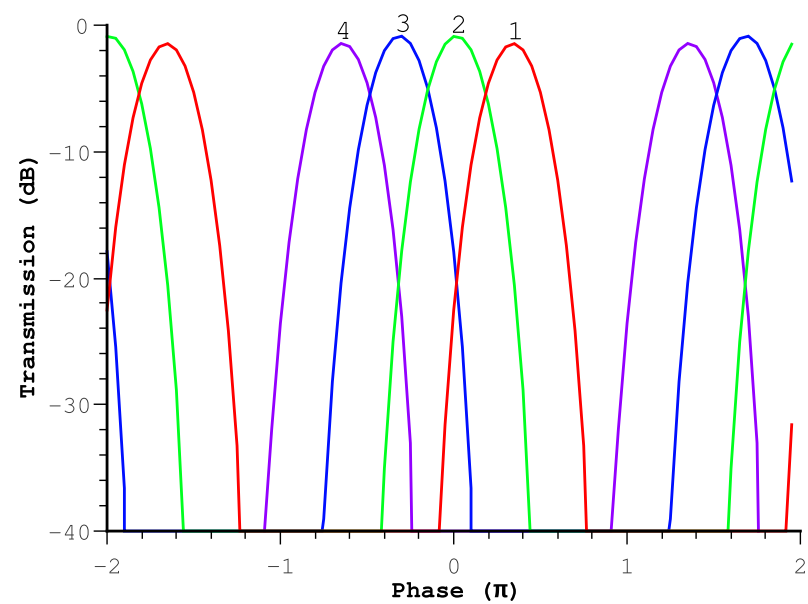

Fig. 3. Simulation of the $1 \times 4$ switch with 12 modulators in the array.

Figure 3 shows the simulation of the $1 \times 4$ switch at 1.55 $\mu \mathrm{m}$. We consider only the waveguide loss, not the additional modulator loss. The simulation shows four operation states where one channel gets maximum power while the other three are minimized. State 2 and 3 experience $-0.9 \mathrm{~dB}$ insertion loss and for the other two states ( 1 and 4$)$ this loss increases to $-1.5 \mathrm{~dB}$. This nonuniformity is similar to that of an AWG wavelength mux/demux [4]. The simulated extinction ratio is $-18 \mathrm{~dB}$, assuming ideal phase control of each modulator.

\section{EXPERIMENT}

The devices were fabricated in IMEC's silicon photonics platform using the ePIXfab multi-project wafer service. We characterize transmission using fiber grating couplers. An array of 20 probes is used to drive the 12 modulators, using the first and last pads as common grounds. The modulators are driven by a NI-PXI analog output card which connected to a PXI chassis. Voltage can be individually controlled between $\pm 10 \mathrm{~V}$, with a current limit of $20 \mathrm{~mA}$.

\section{A. Modulator characteristic}

The modulators are characterized individually with fixed input and output fiber. The transmission is then influenced by the phase induced in the modulated arm. The intensity variation depends on the fraction of the total power in the corresponding arm: the center modulators will induce a larger power change than the outer modulators. Fig. 4 shows that the 6 th modulator has significantly more impact than the $1 \mathrm{st}$ modulator, and the trace of the 6th modulator confirms that the $3.2 \mathrm{~mm}$ long modulator can induce a $2 \pi$ phase shift.

\section{B. Switch}

The switching states can be measured using two methods: by initially calibrating all the modulators, or optimize all drive voltages for each switch state. An accurate calibration of the modulator is difficult as the phase change is not linear with voltage, and the response of the outer modulators is not very pronounced in the overall transmission. Instead, we optimized every modulator for each state. Figure 5 shows the four switching states of the $1 \times 4$ switch.

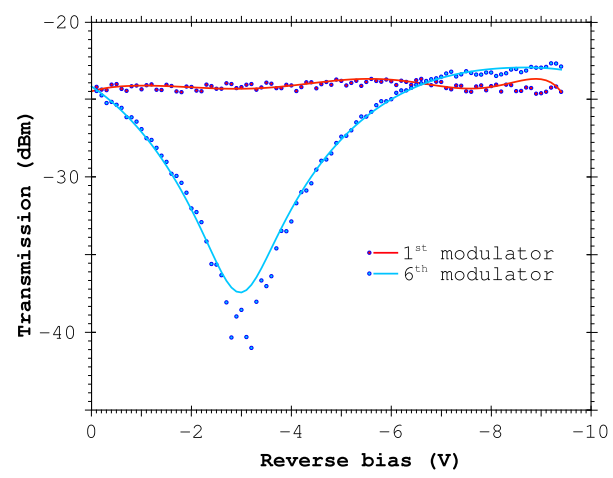

Fig. 4. Optical power variation induced by the (a) $1^{\text {st }}$ and (b) $6^{\text {th }}$ modulator.

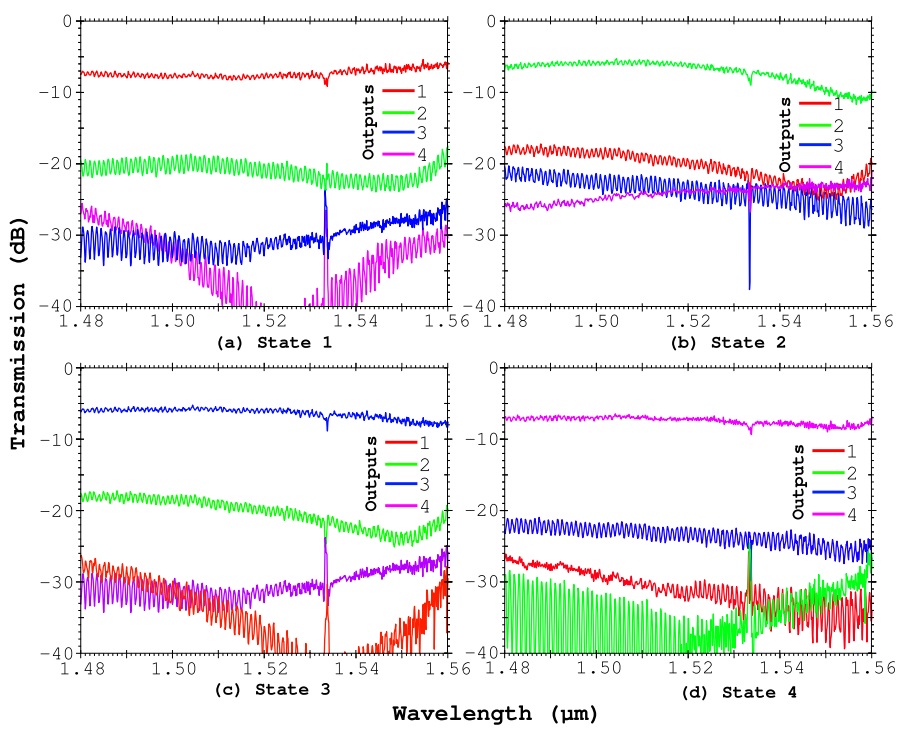

Fig. 5. Measured spectra of the $1 \times 4$ switch for the 4 switching states.

The measurement results show a good agreement with simulations. The additional loss is introduced by the modulators and the extinction is limited by the number of arms in the array. Also, there are some reflections at the modulator interfaces that cause the oscillations in the spectrum. This interface needs to be optimized for lower reflection. Fig. 4 also shows that the modulators have to be driven very close to breakdown to obtain a $2 \pi$ phase change.

\section{CONCLUSION}

We demonstrated an optical switch using a zeroth order AWG and carrier-depletion based silicon modulators. The measured insertion loss of the $1 \times 4$ silicon switch is -6.0 $\mathrm{dB}$ and the crosstalk is better than $-13 \mathrm{~dB}$.

\section{REFERENCES}

[1] T. Tanemura, M. Takenaka, A. Al Amin et al., Photon. Technol. Lett., vol. 20, no. 12, pp. 1063-1065, June 2008.

[2] Y. Hui, M. Pantouvaki, J. Van Campenhout, D. Korn et al., Opt. Express, vol. 20, no. 12, pp. 12926-12 938, Jun 2012.

[3] W. Bogaerts, S. Selvaraja, P. Dumon et al., J. Sel. Top. Quantum Electron., vol. 16, no. 1, pp. $33-44,2010$.

[4] S. Pathak, M. Vanslembrouck, P. Dumon et al., J. Lightwave Technol., vol. 31, no. 1, pp. 87-93, 2013. 